\title{
Retirada Precoce de Introdutor Arterial Guiada pelo Tempo de Coagulação Ativada após Intervenção Coronária Percutânea
}

\author{
Marco Tulio Zanettini ${ }^{1}$, Elias José Perin Conti ${ }^{1}$, Carlos Antônio Mascia Gottschall ${ }^{1}$
}

\section{RESUMO}

Introdução: O tempo de coagulação ativada (TCA) após intervenção coronária percutânea (ICP) femoral pode ser utilizado para guiar a retirada precoce do introdutor arterial. Este estudo comparou os tempos de permanência do introdutor arterial e de repouso após a retirada do introdutor arterial, com e sem controle de TCA, em pacientes que realizaram ICP femoral. Métodos: Ensaio clínico randomizado com 78 pacientes submetidos a ICP femoral eletiva, alocados para retirada do introdutor arterial guiada pelo TCA (grupo 1) ou remoção desse dispositivo sem controle de TCA (grupo 2). No grupo 1, o introdutor arterial foi retirado quando o TCA medido era $\leq 180$ segundos. Após a retirada do introdutor arterial, os pacientes permaneceram por mais 6 horas em repouso absoluto nos dois grupos. Resultados: A média de idade foi de $60 \pm 9$ anos e $57 \%$ dos pacientes eram homens. Os grupos foram semelhantes quanto a características clínicas, complexidade das lesões coronárias, calibre do introdutor arterial, número e tipo de stents utilizados, fármacos empregados e sucesso do procedimento. Os tempos de permanência do introdutor arterial $(95 \pm 35$ minutos vs. $240 \pm 16$ minutos; $\mathrm{P}<0,01)$ e de repouso absoluto $(454 \pm 33$ minutos vs. $600 \pm 12$ minutos; $P<0,01)$ foram significativamente menores no grupo 1. Conclusões: A retirada do introdutor arterial guiada pelo TCA em pacientes submetidos a ICP femoral foi mais precoce e proporcionou redução do tempo total de repouso em comparação à estratégia padrão da instituição.

DESCRITORES: Angioplastia. Stents. Artéria femoral. Coagulação sanguínea.

\footnotetext{
1 Instituto de Cardiologia do Rio Grande do Sul - Fundação Universitária de Cardiologia (IC-FUC) - Porto Alegre, RS, Brasil.

Correspondência: Marco Tulio Zanettini. Rua Bento Gonçalves, 2.048 - 2o pavimento - Centro - Caxias do Sul, RS, Brasil CEP 95020-412

E-mail: marcotz@eletrocorcardiologia.com.br

Recebido em: 25/9/2011 • Aceito em: 6/12/2011
}

ABSTRACT

\section{Early Arterial Sheath Removal Guided by Activated Clotting Time after Percutaneous Coronary Intervention}

Background: Activated clotting time (ACT) can be used for early arterial sheath removal after femoral-approach percutaneous coronary intervention $(\mathrm{PCl})$. This study compared the time for arterial sheath removal, with and without ACT guidance, and bed rest after PCI. Methods: Randomized clinical trial including 78 patients submitted to elective $\mathrm{PCl}$, allocated for ACT-guided (group 1) and non-ACT-guided (group 2) arterial sheath removal. In group 1, the sheath was removed when ACT was $<180$ seconds. After removal of the arterial sheath, patients remained at bed rest for 6 hours in both groups. Results: Mean age was $60 \pm 9$ years and $57 \%$ of the patients were men. Groups were similar for clinical characteristics, coronary lesion complexity, arterial sheath caliber, number and type of stents used, drugs used and success rates. The time for arterial sheath removal $(95 \pm 35$ minutes vs. $240 \pm 16$ minutes; $\mathrm{P}<0.01)$ and bed rest $(454 \pm 33$ minutes vs. $600 \pm 12$ minutes; $P<0.01$ ) were significantly lower in group 1. Conclusions: Arterial sheath removal guided by ACT in patients undergoing $\mathrm{PCl}$ was performed earlier and allowed a reduction of the overall time of bed rest when compared to the institutional standard procedure.

KEY-WORDS: Angioplasty. Stents. Femoral artery. Blood coagulation.

\footnotetext{
A intervenção coronária percutânea (ICP), técnica de revascularização miocárdica mais empregada no tratamento da cardiopatia isquêmica ${ }^{1-4}$, é realizada sob anticoagulação e antiagregação plaquetária combinadas e a heparina não-fracionada (HNF) é, em nosso meio, o anticoagulante mais utilizado nesse cenário. A ação antitrombótica da HNF pode ser monitorizada na sala de hemodinâmica pelo tempo de coagulação ativada (TCA). Preconiza-se medida de TCA entre 250 segundos e 350 segundos para a realização da ICP e $<200$ segundos para a retirada do introdutor arterial. ${ }^{5-11}$
} 
Grande parte dos serviços de hemodinâmica, no entanto, não utiliza rotineiramente o TCA após a ICP para guiar a retirada do introdutor arterial e os pacientes podem permanecer internados por período mais prolongado que o necessário. Essa estratégia acrescenta gastos, reduz o número de pessoas atendidas e gera desconforto ao paciente. ${ }^{12}$

A retirada precoce do introdutor arterial de pacientes submetidos a ICP eletiva pela via femoral guiada pelo TCA pode reduzir o tempo total de repouso, minimizar o desconforto dos pacientes, aumentar a rotatividade dos serviços de hemodinâmica e diminuir custos. O objetivo deste estudo foi comparar os tempos de permanência do introdutor arterial e de repouso após a retirada do introdutor arterial, com e sem controle de TCA, em pacientes que realizaram ICP femoral.

\section{MÉTODOS}

Foi realizado ensaio clínico randomizado com 78 indivíduos submetidos a ICP femoral eletiva no Instituto de Cardiologia do Rio Grande do Sul/Fundação Universitária de Cardiologia (IC-FUC), no período de outubro de 2009 a janeiro de 2010. Esses indivíduos foram subdivididos em dois grupos: grupo 1 (intervenção), em que a retirada do introdutor arterial foi feita sob controle do TCA; e grupo 2 (controle), em que a retirada do introdutor arterial foi realizada 4 horas após o término da ICP (Figura 1). Nos dois grupos, os pacientes permaneciam posteriormente em repouso absoluto, em decúbito dorsal, por outras 6 horas.

Os critérios de inclusão compreendiam pacientes entre 18 anos e 80 anos de idade, de ambos os sexos,

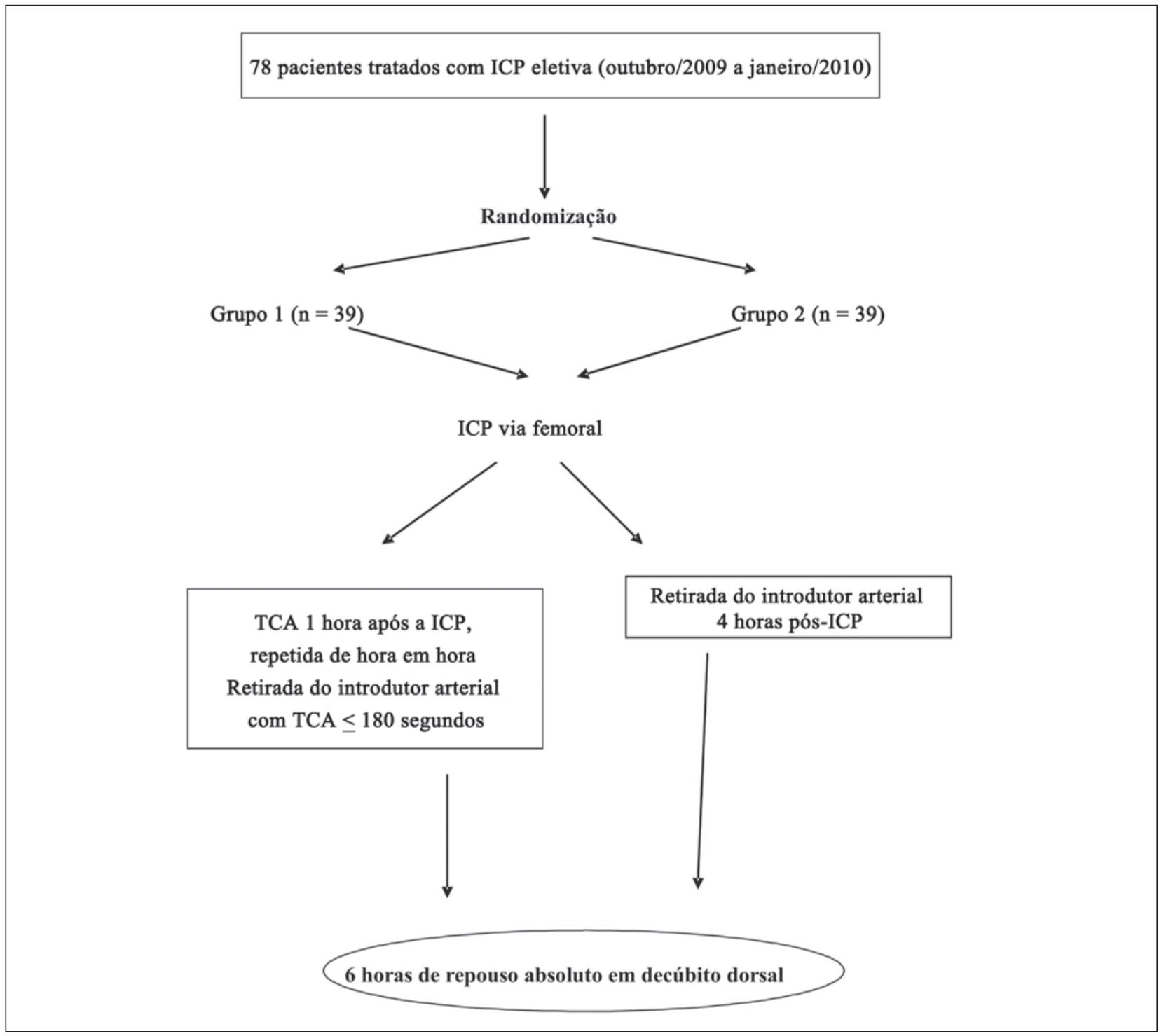

Figura 1 - Desenho do estudo. ICP = intervenção coronária percutânea; $\mathrm{n}=$ número de pacientes; $\mathrm{TCA}=$ tempo de coagulação ativada. 
encaminhados ao serviço de cardiologia intervencionista da instituição para revascularização percutânea eletiva. Foram excluídos os pacientes submetidos a ICP de urgência ou emergencial.

Os pacientes que não estavam em uso prévio de antiplaquetários receberam dose de ataque de $200 \mathrm{mg}$ de ácido acetilsalicílico e $300 \mathrm{mg}$ de clopidogrel, pelo menos 24 horas antes do procedimento. Utilizou-se HNF durante a ICP em todos os participantes, na dose de $70 \mathrm{UI} / \mathrm{kg}$ a $100 \mathrm{UI} / \mathrm{kg}$, não sendo utilizados inibidores da glicoproteína Ilb/IIla nessa população.

No grupo 1, o TCA foi medido 1 hora após o término do procedimento e repetido a cada 60 minutos até que alcançasse valor $\leq 180$ segundos, quando era permitida a retirada do introdutor arterial e a hemostasia obtida por compressão manual. Utilizou-se o dispositivo MCA-2000 ${ }^{\mathrm{TM}}$ (Fundação Adib Jatene - Brasil) para as dosagens de TCA, retirando-se $2 \mathrm{ml}$ de sangue do introdutor arterial. No grupo 2, os pacientes foram tratados conforme a rotina da instituição. Nesse grupo, não eram obtidas medidas de TCA após o procedimento e a hemostasia por compressão manual era realizada 4 horas após o término do procedimento.

As ICPs foram realizadas de acordo com as diretrizes atuais ${ }^{3,13,14}$ e a estratégia final do procedimento foi deixada a critério do operador.

O sucesso do procedimento foi definido como obtenção de sucesso angiográfico (fluxo final TIMI 3, ausência de dissecções e estenose residual < 30\%), na ausência de óbito, infarto do miocárdio (IM) ou revascularização de urgência do vaso-alvo. O diagnóstico de IM foi baseado no aparecimento de nova onda $Q$ patológica em > 2 derivações contíguas no eletrocardiograma e/ou elevação da creatina quinase fração $\mathrm{MB}$ (CK-MB) massa > 3 vezes o limite superior da normalidade. ${ }^{3}$

Sangramento maior foi definido como aquele associado à instabilidade hemodinâmica, requerendo intervenção e/ou necessidade de transfusão de concentrado de hemácias. Sangramento moderado incluiu os hematomas com diâmetro $>5 \mathrm{~cm}$ não associados a instabilidade hemodinâmica. Sangramento menor foi definido por hematoma $\leq 5 \mathrm{~cm}$ sem instabilidade.

As complicações vasculares não-hemorrágicas incluíram pseudoaneurismas, dissecções arteriais e fístulas arteriovenosas. As demais complicações menores registradas foram reação vasovagal e retenção urinária.

A equipe de campo foi constituída por dois médicos que participaram da ICP e foram responsáveis pela randomização, coleta de dados, termo de consentimento, medida do TCA e retirada manual do introdutor arterial.

O projeto do estudo foi aprovado pelo comitê de ética em pesquisa do IC-FUC sob o registro UP 4376/09.
Os pacientes e/ou familiares responsáveis dos indivíduos selecionados foram esclarecidos sobre os objetivos, assinando termo de consentimento livre e esclarecido ao aceitarem a participação no estudo. Todos os pacientes randomizados aceitaram a participação no estudo. O estudo não recebeu financiamento público ou privado.

\section{Análise estatística}

A amostragem foi calculada para prover um poder de $80 \%$ para detectar diferenças clinicamente relevantes entre os grupos. Considerou-se significativa uma diferença de tempo de 60 minutos entre as duas estratégias na avaliação do desfecho primário.

Foram calculados média e desvio padrão para as variáveis quantitativas. As variáveis quantitativas foram analisadas pela pelo teste $t$ de Student e as qualitativas, pelo teste exato de Fisher e qui-quadrado. Para as variáveis tempo para retirada do introdutor arterial e tempo de repouso, utilizou-se o teste de Hedges para mensurar o tamanho do efeito e avaliar a magnitude da diferença entre os dois grupos.

As variáveis foram tabuladas em planilha Excel e analisadas com o auxílio do programa SPSS (Statistical Package for the Social Sciences).

Foram consideradas estatisticamente significantes as associações com $\mathrm{P} \leq 0,05$, bicaudal.

\section{RESULTADOS}

No total, 78 pacientes do IC-FUC, atendidos pelo Sistema Único de Saúde (SUS) e submetidos a ICP eletiva, foram randomizados de outubro de 2009 a janeiro de 2010. Esses indivíduos, subdivididos em dois grupos (grupo 1 e grupo 2, previamente descritos), permaneceram 6 horas em decúbito dorsal sob repouso absoluto após a retirada do introdutor arterial.

A média de idade dos indivíduos era de 59,5 \pm 9,4 anos vs. $61,3 \pm 9,7$ anos $(P=0,41)$, com predomínio do sexo masculino $(53,8 \%$ vs. $61,5 \%$; $P=0,64)$, igual prevalência de fatores de risco para aterosclerose, índice de massa corporal (IMC) de 28,1 $\pm 3,6 \mathrm{~kg} / \mathrm{m}^{2} \mathrm{vs}$. $28 \pm 4,1 \mathrm{~kg} / \mathrm{m}^{2}(\mathrm{P}=0,9)$, e angina estável como quadro clínico tratado com mais frequência, respectivamente para os grupos 1 e 2 (Tabela 1). Não houve diferença entre o número de lesões $(1,2 \pm 0,7$ lesão vs. 1,1 \pm 0,2 lesão; $P=0,67$ ) ou complexidade das lesões tratadas para os grupos 1 e 2, respectivamente (Tabela 2).

Em relação ao procedimento, a dose de HNF (8.012 \pm 1.434 UI vs. $10.725 \pm 1.799$ UI; $\mathrm{P}=0,35)$ e a utilização de introdutor arterial $7 \mathrm{~F}(17,9 \%$ vs. $23,1 \%$; $\mathrm{P}=0,57$ ) foram semelhantes entre os grupos (Tabela 2). O sucesso do procedimento foi semelhante $(94,9 \%$ vs. $97,4 \%$; $P=0,55)$ e não ocorreram óbito, IM ou revascularização de urgência nos dois grupos. 
TABELA 1

Características clínicas dos pacientes

\begin{tabular}{|c|c|c|c|}
\hline & $\begin{array}{l}\text { Grupo } 1 \\
(n=39)\end{array}$ & $\begin{array}{c}\text { Grupo } 2 \\
(n=39)\end{array}$ & $\mathbf{P}$ \\
\hline Idade, anos & $59,5 \pm 9,4$ & $61,3 \pm 9,7$ & 0,41 \\
\hline Sexo masculino, n (\%) & $21(53,8)$ & $24(61,5)$ & 0,64 \\
\hline $\mathrm{IMC}, \mathrm{kg} / \mathrm{m}^{2}$ & $28,1 \pm 3,6$ & $28 \pm 4,1$ & 0,90 \\
\hline Hipertensão arterial, n (\%) & $19(50)$ & $21(55,2)$ & 0,58 \\
\hline Diabetes melito, n (\%) & $15(39,4)$ & $13(34,1)$ & 0,86 \\
\hline Tabagismo, n (\%) & $22(57,8)$ & $24(63,1)$ & 0,53 \\
\hline Revascularização prévia*, n (\%) & $8(21)$ & $9(23,6)$ & 0,36 \\
\hline Quadro clínico, n (\%) & & & $>0,99$ \\
\hline Angina estável & $29(74,4)$ & $30(76,9)$ & \\
\hline Angina instável & $10(25,6)$ & $9(23,1)$ & \\
\hline Fração de ejeção > 50\%, n (\%) & $35(89,7)$ & $36(92,3)$ & 0,69 \\
\hline
\end{tabular}

TABELA 2

Variáveis angiográficas e relacionadas ao procedimento

\begin{tabular}{|c|c|c|c|}
\hline & $\begin{array}{c}\text { Grupo } 1 \\
\text { (39 pacientes/42 lesões) }\end{array}$ & $\begin{array}{c}\text { Grupo } 2 \\
\text { (39 pacientes/41 lesões) }\end{array}$ & $\mathbf{P}$ \\
\hline Número de lesões tratadas & $1,2 \pm 0,7$ & $1,1 \pm 0,2$ & 0,67 \\
\hline Tipo de lesão (AHA/ACC), n (\%) & & & 0,93 \\
\hline A & $11(26,8)$ & $12(29,3)$ & \\
\hline B & $19(45,2)$ & $17(41,4)$ & \\
\hline $\mathrm{C}$ & $12(28,6)$ & $12(29,3)$ & \\
\hline Stent convencional, n (\%) & $35(94,6)$ & $37(97,4)$ & 0,54 \\
\hline Número de stents utilizados & $1,37 \pm 0,31$ & $1,11 \pm 0,69$ & 0,045 \\
\hline Comprimento do stent, mm & $15,9 \pm 5,4$ & $17,5 \pm 5,6$ & 0,24 \\
\hline Diâmetro do stent, mm & $3,04 \pm 0,46$ & $3,05 \pm 0,39$ & 0,89 \\
\hline Heparina não-fracionada, UI & $8.012 \pm 1.434$ & $10.725 \pm 1.799$ & 0,35 \\
\hline Introdutor arterial 7 F, n (\%) & $7(17,9)$ & $9(23,1)$ & 0,57 \\
\hline Tempo de procedimento, minutos & $44 \pm 17,5$ & $38 \pm 13,5$ & 0,09 \\
\hline Sucesso do procedimento, n (\%) & $37(94,9)$ & $38(97,4)$ & 0,55 \\
\hline
\end{tabular}

UI = unidades internacionais.

Foram realizadas, em média, duas dosagens de TCA para cada paciente do grupo 1. O tempo decorrido para a retirada do introdutor arterial foi significativamente menor no grupo $1(95 \pm 35$ minutos vs. $240 \pm$ 16 minutos; $P<0,01)$, proporcionando redução significativa do tempo total de repouso $(454 \pm 33$ minutos vs. $600 \pm 12$ minutos; $P<0,01$ ) (Tabela 3). Na Figura 2 pode-se observar o tamanho do efeito e a magnitude da diferença entre os grupos para os tempos para retirada do introdutor arterial e de repouso total.

Quanto às complicações menores, ocorreram 1 hematoma sem repercussão hemodinâmica com diâ- metro $\leq 5 \mathrm{~cm}$ e 1 reação vasovagal revertida com hidratação rápida e uso de atropina no grupo 1 . No grupo 2, observaram-se 4 hematomas com diâmetro $\leq 5 \mathrm{~cm}$, também sem repercussão hemodinâmica. Outras complicações vasculares não-hemorrágicas não foram observadas.

Todos os pacientes do estudo permaneceram internados por 36 horas.

\section{DISCUSSÃO}

Os pacientes do estudo foram representativos da realidade de um centro cardiológico terciário do sis- 
TABELA 3

Desfechos pós-intervenção coronária percutânea

\begin{tabular}{lccc}
\hline & $\begin{array}{c}\text { Grupo 1 } \\
(\mathbf{n = 3 9 )}\end{array}$ & $\begin{array}{l}\text { Grupo 2 } \\
(\mathbf{n = 3 9 )}\end{array}$ & $\mathbf{P}$ \\
\hline Retirada do introdutor arterial, minutos & $95 \pm 35$ & $240 \pm 16$ & $<0,01$ \\
Decúbito dorsal, minutos & $454 \pm 33$ & $600 \pm 12$ & $<0,01$ \\
Sangramento, $\mathrm{n}(\%)$ & & & $\mathrm{NA}$ \\
$\quad$ Maior ou moderado & 0 & 0 & 0,09 \\
$\quad$ Menor & $1(2,6)$ & 0 & 0,82 \\
Reação vasovagal & $1(2,6)$ & & \\
\hline
\end{tabular}

NA = não-aplicável.

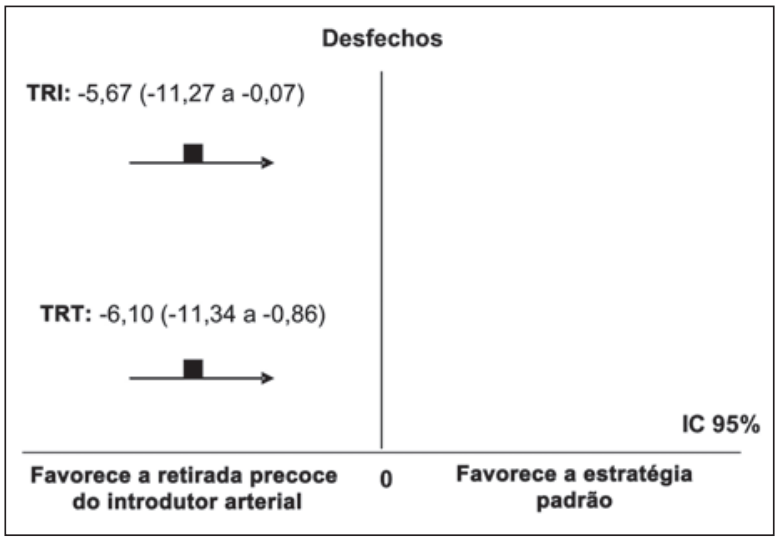

Figura 2 - Tamanho do efeito e intervalos de confiança dos desfechos do estudo. IC 95\% = intervalo de confiança de 95\%; TRI = tempo para a retirada do introdutor arterial; TRT $=$ tempo de repouso total.

tema público brasileiro. Os participantes, em sua maioria, eram homens de meia-idade, com angina estável, portadores de fatores de risco clássicos associados a aterosclerose e de lesões coronárias de baixa e média complexidades. Esses indivíduos receberam adequado manejo antitrombótico e foram tratados predominantemente com um stent convencional, apresentando alto índice de sucesso e baixa incidência de complicações. Este estudo demonstrou redução significativa do tempo de permanência do introdutor arterial e do tempo total de repouso em decúbito dorsal de pacientes que utilizaram o TCA para monitorizar a anticoagulação sistêmica pós-ICP eletiva por via femoral.

Os resultados reforçam os achados de estudos realizados em diversas instituições quanto a remoção precoce do introdutor arterial, redução do tempo de repouso e possibilidade de deambulação precoce de pacientes estáveis submetidos a ICP eletiva. Essa estratégia pode aumentar a disponibilidade de recursos, proporcionando maior conforto ao paciente com diminuição da demanda reprimida do sistema público de saúde. ${ }^{15-18}$

O alto índice de sucesso e a baixa incidência de complicações apresentados no presente estudo são concor- dantes com a literatura vigente e refletem a grande experiência do serviço e de seus operadores, associada à disponibilidade de materiais contemporâneos e com excelente perfil de segurança. ${ }^{19}$ Neste estudo, a estratégia de retirada precoce do introdutor arterial, com redução do tempo de repouso, mostrou-se segura e de baixa complexidade, podendo ser incorporada à prática clínica diária.

\section{Limitações do estudo}

Dentre as limitações do estudo estão a casuística reduzida com consequente limitação estatística e o caráter não-cego do presente ensaio, decorrentes da limitada logística disponível. Além disso, a pequena variabilidade dos tempos de retirada do introdutor arterial e de repouso no grupo 2 limitou a análise estatística. Esses viéses não invalidam, entretanto, os resultados do estudo. Não dispomos de dados a respeito do conforto dos pacientes estudados e dos custos dispendidos por paciente, considerando gastos com material, pessoal e internação. Acreditamos, no entanto, que a estratégia abordada no presente estudo possa gerar maior conforto e diminuição de custos, considerando a possível redução da permanência hospitalar e o aumento da rotatividade do serviço de hemodinâmica.

\section{CONCLUSÕES}

A retirada do introdutor arterial guiada pelo TCA, um marcador objetivo do grau de anticoagulação sistêmica, foi mais precoce e proporcionou redução do tempo total de repouso de pacientes submetidos a ICP eletiva pela via femoral. Essa abordagem foi de fácil aplicabilidade e segura na amostra de pacientes avaliada. Estudos com grande amostragem poderão confirmar esses resultados, contribuindo para o aprimoramento dos cuidados pós-ICP e racionalização de recursos do sistema de saúde.

\section{AGRADECIMENTO}

Agradecemos ao IC-FUC, que tem no estímulo à geração e à divulgação de conhecimento científico uma de suas marcas registradas. 


\section{CONFLITO DE INTERESSES}

Os autores declaram não haver conflito de interesses relacionado a este manuscrito.

\section{REFERÊNCIAS}

1. Piegas LS, Feitosa G, Mattos LA, Nicolau JC, Rossi Neto JM, Timerman A, et al. IV Diretriz da Sociedade Brasileira de Cardiologia sobre Tratamento do Infarto Agudo do Miocárdio com Supradesnível do Segmento ST. Arq Bras Cardiol. 2009;93 (6 Supl 2):179-264.

2. Van de Werf F, Ardissimo D, Betriu A, Cokkinos DV, Falk E, Fox KA, et al. Management of acute myocardial infarction in patients presenting with ST-segment elevation. The Task Force on the Management of Acute Myocardial Infarction of the European Society of Cardiology. Eur Heart J. 2003;24(1):28-66.

3. Mattos LA, Lemos Neto PA, Rassi A Jr, Marin-Neto JA, Sousa AGMR, Devito FS, et al. Diretrizes da Sociedade Brasileira de Cardiologia - Intervenção Coronária Percutânea e Métodos Adjuntos Diagnósticos em Cardiologia Intervencionista (II Edição - 2008). Arq Bras Cardiol. 2008;91(6 Supl 1):1-58.

4. Sousa AGMR, Mattos LAP, Campos Neto CM, Carvalho HG, Stella FP, Nunes G. Intervenções percutâneas para revascularização do miocárdio no Brasil em 1996 e 1997 comparadas às do biênio 1992-1993. Relatório do Registro CENIC (Central Nacional de Intervenções Cardiovasculares). Arq Bras Cardiol. 1998;70(6):423-30

5. Dumaine R, Borentain M, Bertel O, Bode C, Gallo R, White $\mathrm{H}$, et al. Intravenous low-molecular weight heparins compared with unfractionated heparin in percutaneous coronary intervention: quantitative review of randomized trials. Arch Intern Med. 2007;167(22):2423-30.

6. Lundén $M H$, Bengtson $A$, Lundgren SM. Hours during and after coronary intervention and angiography. Clin Nurs Res. 2006;15(4):274-89.

7. Brener SJ, Moliterno DJ, Lincoff AM, Steinhubl SR, Wolski $\mathrm{KE}$, Topol EJ. Relationship between activated clotting time and ischemic or bleeding complications: analysis of 4 recent randomized clinical trials of percutaneous coronary intervention. Circulation. 2004;110(8):994-8.

8. Chew DP, Bhatt DL, Lincoff AM, Moliterno DJ, Brener SJ, Wolski KE, et al. Defining the optimal activated clotting time during percutaneous coronary intervention: aggregate results from 6 randomized controlled trials. Circulation. 2001;103(7): 961-6.

9. Galli A, Palatnik AM. What is the proper activated clotting time (ACT) at which to remove a femoral sheath after PCI? What are the best "protocols" for sheath removal? Ask the experts. Crit Care Nurse. 2005;25(2):88-92.
10. Baim DS. Percutaneous balloon angioplasty and general coronary intervention. In: Baim DS. Grossman's cardiac catheterization, angiography and intervention. Philadelphia: Lippincott; 2006. p. 433-66.

11. Levine GN, Kern MJ. Complications of percutaneous coronary interventions. In: Kern MJ. The interventional cardiac catheterization handbook. Pennsylvania: Mosby; 2004. p. 162-84.

12. Lauck S, Johnson JL, Ratner PA. Self-care behavior and factors associated with patient outcomes following same-day discharge percutaneous coronary intervention. Eur J Cardiovasc Nurs. 2009;8(3):190-9.

13. Kushner FG, Hand $M$, Smith SC Jr, King SB 3rd, Anderson JL, Antman EM, et al. 2009 Focused Updates: ACC/AHA Guidelines for the Management of Patients With ST-Elevation Myocardial Infarction (Updating the 2004 Guideline and 2007 Focused Update) and ACC/AHA/SCAI Guidelines on Percutaneous Coronary Intervention (updating the 2005 Guideline and 2007 Focused Update): a report of the American College of Cardiology Foundation/American Heart Association Task Force on Practice Guidelines. Circulation. 2009;120(22): 2271-306.

14. Gubolino LA, Mangione JA, Silva SS, Marin-Neto JA, Lopes MACQ, Salvador Junior D, et al. Diretrizes da Sociedade Brasileira de Hemodinâmica e Cardiologia Intervencionista sobre Qualidade Profissional e Institucional, Centro de Treinamento e Certificação Profissional em Hemodinâmica e Cardiologia Intervencionista. Rev Bras Cardiol Invasiva. 2008; 16(3 Supl 3):8-32.

15. Keeling AW, Fisher CA, Haugh KH, Powers ER, Turner MS Reducing time in bed after percutaneous transluminal coronary angioplasty (TIBS III). Am J Crit Care. 2000;9(3):185-7.

16. Lasevitch R, Melchior R, Gomes V, Caramori PA, Roman M, Alcalde $R$, et al. Early discharge using five french guiding catheter for transfemural coronary stenting: a feasibility and safety study (EDU 5Fr Study). Am J Cardiol. 2005;96(6): 766-8.

17. Tengiz I, Ercan E, Bozdemir H, Durmaz O, Gurgun C, Nalbantgil I. Six hour ambulation after elective coronary angioplasty and stenting with $7 \mathrm{~F}$ guiding catheters and low dose heparin. Kardiol Pol. 2003;58(2):93-7.

18. Augustin AC, Quadros AS, Sarmento-Leite R. Early sheath removal and ambulation in patients submitted to percutaneous coronary intervention: a randomised clinical trial. Int J Nurs Stud. 2010;47(8):939-45

19. Abelin AP, Quadros AS, Zanettini MT, Leboute FC, Yordi LM, Cardoso CR, et al. Doze anos de experiência com implante de stents coronários em 5.284 pacientes. Rev Bras Cardiol Invasiva. 2009;17(3):346-51. 